\title{
Association of Spasmolytic Polypeptide-Expressing Metaplasia with Carcinogen Administration and Oxyntic Atrophy in Rats
}

\author{
Hirokazu Yamaguchi, James R. Goldenring, Michio Kaminishi, and Jeffrey R. Lee \\ Department of Gastrointestinal Surgery (HY, MK), Graduate School of Medicine, University of Tokyo, Tokyo, Japan; \\ Augusta Veterans Affairs Medical Center, Departments of Medicine, Surgery, and Cellular Biology and Anatomy (JRG) \\ and Pathology (JRL), Institute of Molecular Medicine and Genetics, Medical College of Georgia, Augusta, Georgia
}

SUMMARY: Spasmolytic polypeptide (TFF2)-expressing metaplasia (SPEM) is a gastric metaplastic lineage associated with the development of intestinal-type gastric adenocarcinoma. To study the etiology of this potential neoplastic precursor metaplasia, we used surgical rat models of remnant gastric adenocarcinoma studied with and without exposure to nitroso carcinogen. Animals with truncal vagotomy without duodenogastric reflux procedures demonstrated normal mucous neck cell spasmolytic polypeptide (SP) immunostaining. In these animals, anti-proliferating cell nuclear antigen (PCNA)-labeled nuclei were found in the normal midgland progenitor zone. Rats that received anatomic alterations that augmented the degree of duodenogastric reflux, however, revealed expansion of basally placed SP immunoreactive cells with early phenotypic changes of SPEM. Seventy percent of animals with antrectomy and carcinogen (with or without vagotomy) developed SPEM at the base of the gastric mucosa. In association with the appearance of this metaplastic lineage, a distinct second zone of PCNA-labeled nuclei developed in the deepest portion of the mucosa. Of interest, three animals demonstrating these changes developed intestinal-type gastric adenocarcinoma. Finally, we studied the immunostaining pattern of intrinsic factor, normally a chief cell marker in rat fundic mucosa. In animals with SPEM, we observed coexpression of SP and intrinsic factor in SPEM cells at the base of the mucosa. These findings support our hypothesis that SPEM develops from a second progenitor cell population, reflecting either the unmasking of a cryptic zone or transdifferentiation of chief cells. (Lab Invest 2002, 82:1045-1052).

$T$ he postgastrectomy stump is still considered at risk for the development of remnant gastric adenocarcinoma (Kaminishi et al, 1995). A spectrum of causative factors have been investigated, including alkaline duodenogastric reflux (bile reflux gastritis), intragastric bacterial flora overgrowth with an increase in carcinogenic $\mathrm{N}$-nitroso compounds, denervation, and decreased vascularity (Kaminishi et al, 1997). In recent years, indications for gastrectomy for peptic ulcer have decreased, and the long-term survival rates after gastrectomy for gastric cancer have increased (Kaminishi et al, 1996). This improved survival is due to advances in the surveillance, diagnosis, and treatment of this deadly disease (Kaminishi et al, 1996). As a result, the incidence of remnant gastric cancer after curative surgery for gastric cancer has increased (Furukawa et al, 1993).

The characteristics of remnant gastric cancer differ according to the primary disease at the time of gas-

DOI: 10.1097/01.LAB.0000022225.45996.21

Received March 26, 2002.

This work was supported by Department of Veterans Affairs Merit Review awards to both JRL and JRG.

Address reprint requests to: Dr. Jeffrey R. Lee, Chief, Pathology and Laboratory Medicine Department, Veterans Affairs Medical Center, PLMD (239), One Freedom Way, Augusta, Georgia 30904. E-mail: jlee@mail.mcg.edu trectomy. The time interval between primary gastrectomy and development of remnant gastric cancer is shorter in cases of gastrectomy for malignancy (Kaminishi et al, 1997). Patients undergoing resection for benign disease tend to develop diffuse type malignancies at the anastomotic site, whereas after malignant resections a higher percentage of intestinal-type malignancies in the gastrectomized stump or gastric body is observed (Kaminishi et al, 1996).

Rodent remnant gastric cancer models have assisted in the explanation of this phenomenon. Animals with surgically created duodenogastric reflux without carcinogenic initiation developed diffuse type remnant gastric carcinomas at the anastomotic site, similar to the pattern in human patients after gastrectomy for benign disease (Kaminishi et al, 1997). However, rodents with identical postsurgical duodenogastric reflux that were pretreated with nitrite carcinogen demonstrated a higher percentage of intestinal type stump and body carcinomas, correlating with the pattern in humans after resections for malignant disease (Kaminishi et al, 1996, 1997). In the benign model, remnant gastric cancers developed at the anastomotic site, where the influence of pancreaticobiliary reflux was prolonged. However, it appears that the body mucosa of the rodents pretreated with carcinogen harbored changes of malignant initiation. These phenomena provide an explanation for the early malignant 
changes and the development of carcinoma remote from pancreaticobiliary reflux.

Recently, we reported on a cohort of Japanese patients with remnant gastric cancer (Yamaguchi et al, 2002). In that study, we observed spasmolytic polypeptide (SP)-expressing metaplasia (SPEM) in the surrounding mucosa in $88 \%$ of remnant cancers. In addition, we identified SP staining within dysplastic cells in $67 \%$ of the cases and within malignant cells in $25 \%$ of invasive malignancies. We have previously described SPEM in mice infected with Helicobacter felis (Wang et al, 1998), as well as in humans with fundal predominant $H$. pylori gastritis and in the mucosa surrounding gastric adenocarcinoma (Schmidt et al, 1999). The cells of this lineage recapitulated the morphology of duodenal Brunner's glands and were noted in the fundic mucosa of $65 \%$ of patients infected with $H$. pylori. Further, this metaplastic lineage was identified in $91 \%$ of gastric resections for adenocarcinoma, and SP immunostaining was seen in dysplastic cells in $59 \%$ of cases. These studies suggested that SPEM might represent a precursor to gastric adenocarcinoma.

In this study, we used a well-established postgastrectomy rodent animal model (Kaminishi et al, 1995, 1996, 1997) to characterize further the etiology of SPEM. SPEM developed in the setting of gastrectomy and nitrite carcinogen induction. In addition, and coincident with the development of SPEM, anti-proliferating cell nuclear antigen (PCNA) immunoreactive cells appeared at the base of the fundic glands. Cells of the SPEM lineage were also dually immunoreactive for SP and intrinsic factor. The data indicate that SPEM emanates either from the unmasking of a cryptic mucosal progenitor zone or from transdifferentiation of gastric chief cells.

\section{Results}

\section{Histology}

As detailed below, six groups of animals were examined. Groups I and II received truncal vagotomy without or with duodenogastric reflux. Groups III and IV received antrectomy and Bilroth I anastomoses without or with truncal vagotomy. Groups $\mathrm{V}$ and $\mathrm{VI}$ received the nitrite carcinogen $\mathrm{N}$-methyl-N'-nitro-Nnitrosoguanidine (MNNG) treatment before



Figure 1.

Number of parietal cells per gland for each group. Control versus * $p<0.01$, ${ }^{*}$ versus ${ }^{* \star} p<0.05,{ }^{*}$ versus ${ }^{* \star *} p<0.01$.

antrectomy and Bilroth I anastomosis without or with truncal vagotomy. A final seventh group of untreated rats were also examined. Oxyntic atrophy, defined as significant loss of parietal cell mass, was quantified in untreated control animals and in each resected stomach. Progressive oxyntic atrophy correlated with the severity of operative procedure and addition of carcinogen (Fig. 1). There was an approximate $22 \%$ decrease in parietal cells from the control group to groups I and II $(p<0.01)$. There was an approximate $23 \%$ decrease in parietal cell mass from groups I and II to groups III and IV ( $p<0.05)$, and a $40 \%$ decrease from groups I and II to groups V and VI ( $p<0.01$ ). Gastritis cystica profunda (adenocystic change) was not seen in groups I and II, but was identified in the more severe surgical groups $(p<0.003)$. Intestinal metaplasia was not seen in groups I to IV, but appeared in $17 \%$ of Group V and $25 \%$ of Group VI. Adenocarcinoma was not identified in these postoperative rats unless carcinogen was administered $(p<$ $=0.05$ ) (Table 1).

\section{Immunohistochemistry}

Immunohistochemistry was performed with a mAb specific for SP (TFF2). The truncal vagotomy animals demonstrated normal mucous neck cell immunostain-

Table 1. Characterization of Animal Groups

\begin{tabular}{lrrrrrrr}
\hline & \multicolumn{7}{c}{ Group } \\
\cline { 2 - 8 } & \multicolumn{1}{c}{ I } & II & III & IV & V & VI \\
\hline Number of cases & 7 & 5 & 2 & 3 & 6 & 4 & $p=0.0033$ \\
Gastritis cystica profunda & $0 \%$ & $0 \%$ & $50 \%$ & $67 \%$ & $67 \%$ & $50 \%$ & $n$ ns \\
Intestinal metaplasia & $0 \%$ & $0 \%$ & $0 \%$ & $0 \%$ & $17 \%$ & $25 \%$ & $p=0.041$ \\
Adenocarcinoma & $0 \%$ & $0 \%$ & $0 \%$ & $0 \%$ & $17 \%$ & $50 \%$ & $p=0.0001$ \\
SPEM & $0 \%$ & $0 \%$ & $0 \%$ & $0 \%$ & $50 \%$ & $100 \%$ & $p$ \\
PCNA-labeling & MG & MG & MG & ED & Dual & Dual & \\
\hline
\end{tabular}

SPEM, spasmolytic polypeptide expressing metaplasia; MG, physiologic midgland zone; Dual, midgland and deep zone staining; ED, early dual staining; ns, not significant. 
ing for SP (Fig. 2a). In animals with duodenogastric reflux, we observed scattered SP immunoreactive glands at the base of the mucosa (Fig. 2b). SP immunostaining of the antrectomy without vagotomy Group III demonstrated expansion of the midgland mucous neck cell region (Fig. 2c). The addition of vagotomy caused a marked expansion of basally located SP immunoreactive cells with early phenotypic changes toward SPEM (Fig. 2d). Finally, animals receiving carcinogen in groups $\mathrm{V}$ and $\mathrm{VI}$ developed cells with the phenotype of SPEM at the base of the atrophic oxyntic mucosa (Fig. 2e). Group V demonstrated SPEM in $50 \%$ of cases, whereas Group VI had


Figure 2.

Spasmolytic polypeptide immunostaining in rat models. a, Group I, physiologic staining in mucous neck cells in midgland zone; b, Group II, scattered spasmolytic polypeptide (SP)-positive staining at base of glands; c, Group III, expansion of midgland zone; d, Group IV, expansion of SP staining to base of glands; e, Group VI, development of spasmolytic polypeptide-expressing metaplasia (SPEM) at base of atrophic mucosa. Left, Bar $=100 \mu \mathrm{m}$. Right, Bar $=30 \mu \mathrm{m}$.
SPEM in $100 \%$. These results demonstrated a significant increase in SPEM in rats exposed to carcinogen (Groups III and IV versus Groups V and VI, $p=0.0256$; Groups I to IV versus Groups V and VI, $p=0.0001$ ) (Table 1).

To assess proliferating cells, we performed immunostaining with antibodies against PCNA. In Groups I, II, and III, PCNA-labeled nuclei were observed in the normal progenitor zone, located approximately onethird of the distance between the luminal surface and glandular base (Fig. 3a). With the addition of truncal vagotomy to the antrectomy group, there was widening of the progenitor zone with expansion of PCNAlabeled cells in the midzone of the glands (Fig. 3b). With the addition of carcinogen, two distinct zones of PCNA immunostaining were seen (Fig. 3c). In addition to proliferative activity in cells at the physiologic midgland zone, we observed PCNA staining cells in the deepest portions of the mucosa (Fig. 3c). The distribution of deep gland PCNA staining cells in the atrophic mucosa correlated with the appearance of SPEM at the base of the glands.

\section{SPEM Dual Staining with Spasmolytic Polypeptide and Intrinsic Factor}

Intrinsic factor is secreted from chief cells in the rat fundic mucosa. In rats without MNNG treatment (Groups I to IV), intrinsic factor immunoreactivity was observed in normal appearing chief cells at the base of fundic glands (data not shown). However, in the carcinogen groups ( $\mathrm{V}$ and $\mathrm{VI}$ ), intrinsic factor antibodies stained metaplastic cells at the base of atrophic glands that were also stained by SP antibodies.

To establish more definitely whether SPEM cells in the deep glands also expressed intrinsic factor, double immunofluorescence for SP and intrinsic factor was performed. No SP staining was observed in intrinsic factor-immunoreactive cells in groups I to IV (data not shown). However in the MNNG-treated rats, SPEM cells were immunoreactive for SP and intrinsic factor (Fig. 4), especially at the deepest portion of the glands. The intracellular distribution of SP and intrinsic factor in SPEM cells was distinct, suggesting that these two secreted products were present in different vesicular populations within the cells.

\section{Discussion}

After gastrectomy for benign or malignant disease, the remnant stomach is more susceptible to the development of gastric adenocarcinoma. Although the frequency of resection for benign gastric disease is decreasing, the incidence of remnant gastric cancer has increased (Furukawa et al, 1993). This increase undoubtedly reflects longer survival rates in gastric cancer patients due to advances in aggressive surveillance and treatment programs. In particular, Japanese patients undergoing gastrectomy for early gastric cancer demonstrated an increase in stump carcinomas (Kaminishi et al, 1997). Many causative factors have been implicated in this malignant process, but the 


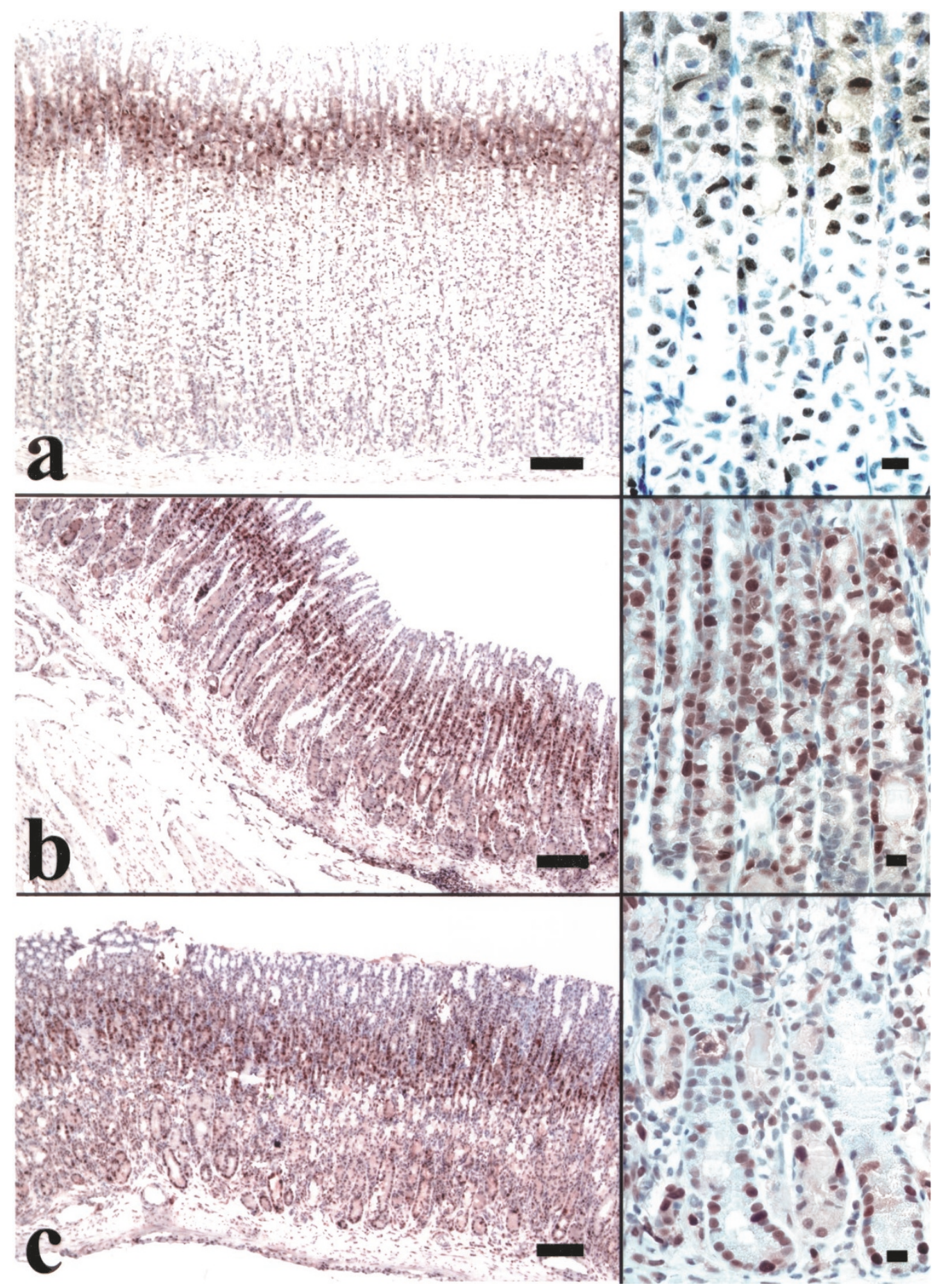

Figure 3.

PCNA immunostaining in rat models. a, Group I, physiologic staining in midgland proliferative zone; b, Group IV, scattered proliferating cells at base of glands; $\mathrm{c}$, Group VI, distinct dual zones of proliferation at midgland and base of glands. Left, Bar $=100 \mu \mathrm{m}$. Right, Bar $=30 \mu \mathrm{m}$.

etiology remains obscure. One explanation includes the progression of a premalignant focus in the remnant stomach.

Transformation of metaplasia to malignancy is a well-known phenomenon within the gastrointestinal tract. Extrapolating from mouse models and human studies, we hypothesized that SPEM may represent a precursor to gastric adenocarcinoma in humans. Recently, we reported on a series of 19 cases of remnant gastric carcinoma from Japan (Yamaguchi et al, 2002). Eighty-eight percent of these cases demonstrated SPEM in the surrounding mucosa, $67 \%$ demonstrated SP staining within dysplastic cells, and $25 \%$ demonstrated SP immunostaining in invasive malignancy. Although carcinogenesis within gastric remnants and in the setting of longstanding Helicobacter sp. infection may involve different etiologies, both processes lead to significant parietal cell loss. To that end, we 

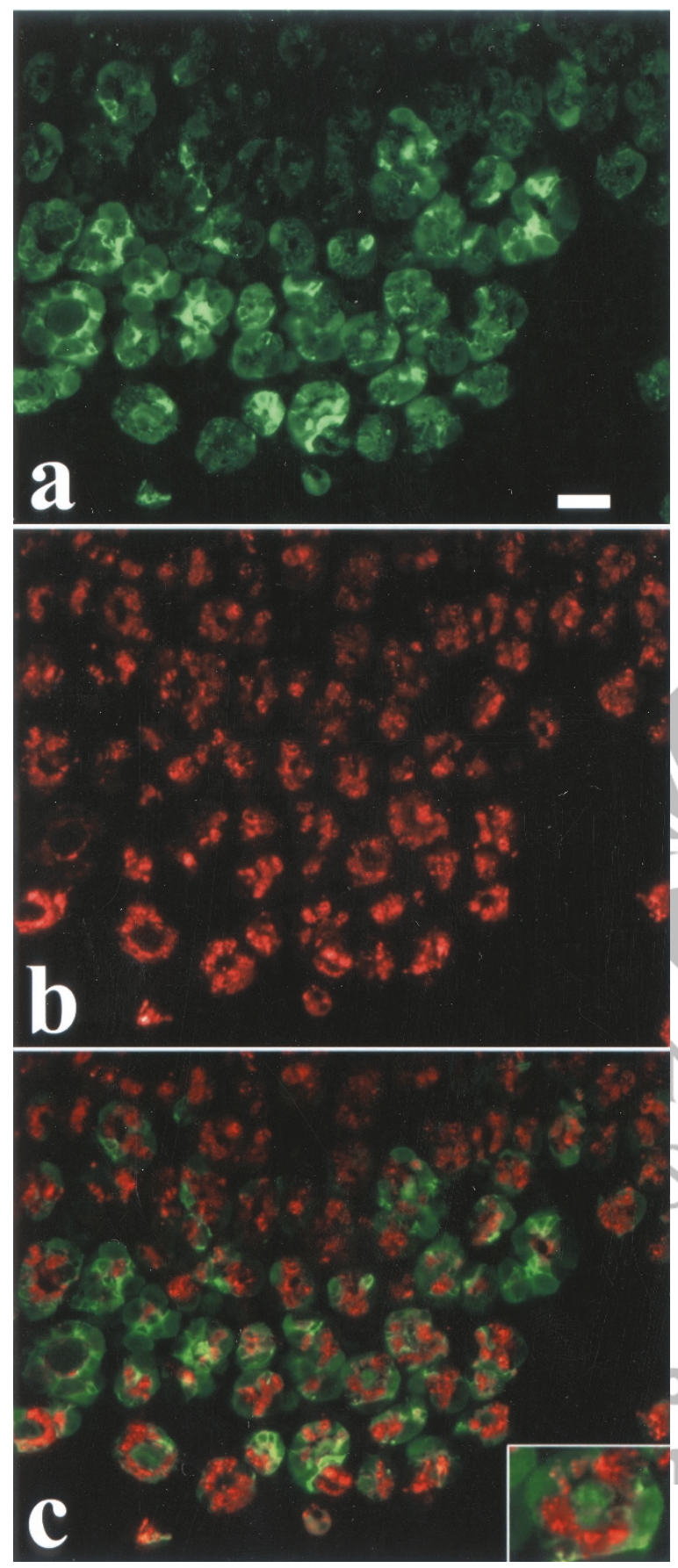

Figure 4.

Expression of intrinsic factor and SP in SPEM cells. Mucosa from an animal treated with $\mathrm{N}$-methyl-N'-nitro-N-nitrosoguanidine (MNNG) was dual stained for intrinsic factor (a, green) and SP (b, red). An overlay of the dual staining (c) demonstrates expression of intrinsic factor and SP within individual SPEM cells without vesicular colocalization (see inset). Bar $=41 \mu \mathrm{m}$.

compared 17 patients with endoscopic and histologic evidence of gastritis secondary to $H$. pylori with 10 patients without gastritis (Schmidt et al, 1999). Sixtyfive percent of the patients with $H$. pylori fundic gastritis demonstrated SPEM, whereas patients without gastritis did not demonstrate this lineage. Next we studied 22 patients who underwent gastric resection for advanced fundal or fundal/antral adenocarcinoma.
Ninety-one percent of these cases demonstrated SPEM within the mucosa surrounding the invasive malignancy. Importantly, we observed immunostaining in dysplastic gastric epithelium or carcinoma in situ in $59 \%$ of the specimens. One resection specimen revealed SP immunostaining within adenocarcinoma.

Experiments involving Wistar rats have provided insights into the development of remnant gastric adenocarcinoma. These investigations have implicated the participation of nitrites, duodenogastric reflux, and truncal vagotomy in this carcinogenesis. The nitrite carcinogen, MNNG, is an initiator and promoter of adenocarcinoma in these remnant cancer rat models. Use of MNNG has successfully induced malignancy in rats with and without gastrectomy with a dose and duration response (Dahm and Werner, 1976; Kondo et al, 1984; Salmon et al, 1985). All of these early studies demonstrated that the length of time required for malignant transformation was significantly shorter in the postgastrectomy animals.

Elegant animal model experiments have demonstrated the participation of duodenogastric reflux in the development of remnant gastric adenocarcinoma. Multiple studies identified malignant growths in rats with duodenogastric reflux without MNNG induction (Langhans et al, 1981; Mason et al, 1988; Mason and Filipe, 1990; Szentleleki et al, 1990). Using a variety of surgical reconstructions after gastrectomy, investigators concluded that the postgastrectomy anatomy with the highest degree of reflux demonstrated the highest rate of remnant carcinomas (Langhans et al, 1981; Mason and Filipe, 1990; Szentleleki et al, 1990). Of note, diversion of all pancreaticobiliary secretions from the gastric mucosa with a Roux-en-Y reconstruction protected ăgainst the development of malignancy. Further, Mason et al (1988) were able to develop animal models that selectively allowed reflux of bile only and bile mixed with pancreatic secretions. Their data revealed that bile alone was not carcinogenic, and that pancreatic exocrine secretions were necessary. It is known that bile salts are ulcerogenic, however, it may be the addition of lysolecithin and/or other byproducts of pancreatic enzymatic digestion that promotes carcinogenesis (Ritchie, 1984).

Further animal experiments by Kaminishi et al (1995, $1996,1997)$ expanded on the etiology of postgastrectomy carcinogenesis. Using the data derived from a Wistar rat model similar to the model outlined in this paper, they made several observations. First, truncal vagotomy induced atrophic gastritis, probably due to decreased defense mechanisms, including alterations in mucosal blood flow, mucin production, and epithelial cellular renewal. In addition, they hypothesized that there were two processes of carcinogenesis, depending on the site of recurrence. Anastomotic remnant cancers showed a diffuse histologic type and were associated with an increased degree of gastritis cystica profunda. In contrast, a higher percentage of intestinal-type malignancies arose in the gastric stump and body, most commonly in the group of rats that received preoperative carcinogen (Kaminishi et al, 1996). 
Animal models have also been instrumental in defining the mucosal changes associated with oxyntic atrophy. Helicobacter felis infection causes chronic gastritis and parietal cell loss in C57BL/6 mice that mimics the pathologic features seen in chronic human gastric infection (Fox et al, 1996; Lee et al, 1990). Using this same animal model, Wang et al (1998) noted that the chronic infection led to a marked decrease in the volume of gastric parietal and chief cells. These cells were replaced with SPEM, a mucous cell metaplasia that emanated from the base of the atrophic mucosa and demonstrated morphology similar to duodenal Brunner's glands. In addition, there was glandular invasion into the submucosa in $40 \%$ of the mice, with development of invasive adenocarcinoma in mice infected for over 9 months (Wang, personal communication).

The phenotypic expressions of two transgenic mouse models further contribute to our understanding of the mucosal changes associated with parietal cell loss. The insulin-gastrin transgenic mouse was engineered to over-express human heptadecapeptide gastrin from the rat insulin I promoter (Wang et al, 2000). After an initial increase, there was a steady decline in parietal cells. This decline was accompanied by the emergence of an SP immunoreactive population of cells at the base of the mucosa. These mice then went on to develop dysplasia and invasive intestinal type adenocarcinoma. Of interest, infection of these mice with $H$. felis markedly accelerated the neoplastic process, presumably from synergistic development of oxyntic atrophy. The second model involves transgenic mice overexpressing TGF $\alpha$, under the control of the metallothionein promoter (MTTGF $\alpha$ ). The mice demonstrated a massive foveolar hyperplasia and parietal cell loss that mimics Ménétrier's disease (Dempsey et al, 1992). In addition, the phenotype of this transgenic mouse demonstrated the appearance of SP-positive cells at the base of the gastric glands. Cells in the same region demonstrated bromodeoxyuridine (BrdU) S-phase immunostaining (with a loss of the physiologic BrdU staining in the midgland fundic progenitor zone), suggesting the unmasking of a progenitor zone that produces SP positive cells.

A fourth animal model of oxyntic atrophy involves the administration to rats of DMP-777, a drug that leads to a reversible pharmacologic ablation of parietal cells (Goldenring et al, 2000). DMP-777 is an orally bioavailable, cell permeant neutrophil elastase inhibitor, and 2 to 4 days after treatment there was extensive parietal cell necrosis. This loss of parietal cells appeared to result from a direct protonophore capacity of the drug toward these cells. In conjunction with the profound parietal cell loss, there was a prominent increase in proliferating cells as evidenced by an increase in BrdU-labeled cells. In addition to increased labeling in the normal progenitor zone, a second BrdU-positive zone emerged at the base of the mucosa. Further, these basally oriented cells corresponded with the emergence of an SP-labeled mucous cell lineage deep in the gastric glands. The novel mucous cell lineage continued to expand throughout 10 days of acute treatment and showed the morphologic and immunochemical staining characteristics of SPEM. These findings supported the hypothesis that SPEM developed from a cryptic progenitor zone that becomes unmasked secondary to parietal cell loss.

This study represents a fifth animal model of oxyntic atrophy with SPEM emergence and basal glandular proliferation. Our data revealed that surgically treated animals demonstrated an increased severity of oxyntic atrophy with each additional anatomic aberration. However, SPEM did not develop at the base of the atrophic oxyntic mucosa until gastrectomy, with or without vagotomy, was coupled with preoperative carcinogen administration. These groups ( $\mathrm{V}$ and $\mathrm{VI}$ ) developed remnant adenocarcinoma with a frequency of $17 \%$ and $50 \%$, respectively. In addition, the pattern and nature of immunostaining confirmed the insights of the origin of SPEM. The appearance of this metaplastic lineage was associated with the emergence of a second deep zone of PCNA-labeling. Associated with this PCNA labeling and SPEM were cells dual labeled with SP and intrinsic factor.

The present studies are the first to demonstrate dual labeling of SPEM cells for both SP and intrinsic factor. In the normal mouse fundus, intrinsic factor is observed exclusively in chief cells, and we have not been able to discern any dual SP and intrinsic factorstained cells. The dual staining of SPEM with SP and intrinsic factor has led to two working hypotheses for the origin of the SPEM lineage. First, as previously suggested (Goldenring et al, 2000), parietal cell loss may lead to the unmasking of a second progenitor zone, which is normally suppressed in the deep gastric oxyntic mucōsa. All other mucosal regions in the gastrointestinal tract, including the gastric antrum, are populated from stem cells in the deepest aspect of the mucosa. With injury and parietal cell loss, this cryptic progenitor zone could be activated and produce SPEM cells to repopulate the atrophic mucosa.

Alternatively, as a second hypothesis, oxyntic atrophy may lead to transdifferentiation of chief cells into the SPEM lineage. Karam and Leblond (1993) have demonstrated that the mouse zymogenic cell lineage is derived from mucous neck cells during their basal migration and maturation. They identified a prezymogenic cell lineage deep to the progenitor zone that was characterized by a heterogeneous population of cytoplasmic secretory granules. These prezymogenic cells mature into chief cells. Thus, vesicular SP and intrinsic factor staining in SPEM cells might accrue from chief cells transdifferentiating into either a proliferative prezymogenic type cell or a basally placed stem cell. Whatever the mechanism, these metaplastic cells then begin dividing and repopulating the atrophic mucosa with SPEM. Whether these SPEM cells can redifferentiate into surface cell elements, as seen in the Ulcer Associated Cell Lineage in small intestine (Wright et al, 1990), remains to be determined.

In summary, SPEM developed most prominently in rats treated with MNNG, the groups with an increased incidence of gastric adenocarcinoma. The SPEM cell 
lineage was immunoreactive for both SP and intrinsic factor. These results support the hypothesis that SPEM develops in the setting of oxyntic atrophy from a novel progenitor cell population reflecting either the activation of a cryptic stem cell lineage or transdifferentiation of chief cells.

\section{Materials and Methods}

\section{Experimental Randomization}

Surgical models, with and without carcinogen, were made on 27 male Wistar rats weighing approximately 200 grams. The stomachs of three nonsurgical rats were evaluated as controls. As an initiator of carcinogenesis, low concentrations of the carcinogenic agent, MNNG (50 mg/l), were given in drinking water to the rats for 10 weeks. After completion of the protocol administration of MNNG or placebo, the rats were assigned into the following groups: Group I, truncal vagotomy; Group II, truncal vagotomy and duodenogastric reflux model (Kaminishi et al, 1987); Group III, antrectomy with Billroth I; Group IV, truncal vagotomy and antrectomy with Billroth I; Group V, antrectomy with Billroth I and preoperative MNNG; Group VI, truncal vagotomy, antrectomy with Billroth I, and preoperative MNNG. There were seven animals in Group I, five in Group II, two in Group III, three in Group IV, six in Group V, and four in Group VI. Briefly, duodenogastric reflux was accomplished by anastomosing the fore stomach to the upper part of the jejunum, 5 to $6 \mathrm{~cm}$ distal to the ligament of Treitz. Two weeks later, the duodenum was transected just distal to papilla of Vater (Kaminishi et al, 1987). Truncal vagotomy was performed at the level of the diaphragm. No carcinogenic agents were given after the surgical procedures.

All rats survived and were killed 30 weeks after surgery by cardiac puncture. After the stomachs were removed, they were opened along the greater curvature and fixed in a 10\% formalin solution for 24 hours. After fixation, the stomachs were dissected into $4-\mathrm{mm}$ wide strips, embedded in paraffin, and cut into $4-\mu \mathrm{m}$ sections. Replicate sections were stained with hematoxylin and eosin (H\&E), as well as periodic acid Schiff (PAS) and alcian blue (AB). Parietal cells were counted on H\&E histochemical slides. Parietal cell numbers in 10 glands from the three control mice and each study mouse were determined and averaged (Fig. 1).

\section{Immunohistochemistry}

For SP immunohistochemistry, deparaffinized replicate sections were blocked with $5 \%$ goat serum in PBS and then incubated with murine monoclonal lgM anti-SP $(1: 50)$ overnight at $4^{\circ} \mathrm{C}$. Indirect immunohistochemical detection was then performed through incubation with biotinylated anti-mouse $\operatorname{lgM}$, streptavidin-conjugated alkaline phosphatase (Vectastain ABC-AP kit; Vector Laboratories, Burlingame, California), and chromagen development with Vector Red substrate solution (Vector Laboratories).
For intrinsic factor and PCNA immunohistochemistry, deparaffinized replicate sections were pretreated with methanol and hydrogen peroxide, and blocked with $5 \%$ goat serum in PBS. Steamer epitope retrieval with citrate buffer (BioGenex Laboratories, San Ramon, California) was performed before immunostaining for intrinsic factor. Sections were incubated with rabbit polyclonal $\lg G$ anti-human intrinsic factor $(1: 3,000)$ for 1 hour at room temperature. For PCNA, sections were incubated with murine monoclonal antihuman IgG PCNA (1:300; Dako, Carpinteria, California) for 30 minutes at room temperature. Indirect immunohistochemical detection was then performed through incubation with biotinylated anti-rabbit IgG streptavidin-conjugated horseradish peroxidase (Vectastain Elite ABC kit; Vector Laboratories) and chromagen development with diaminobenzidine (BioGenex).

\section{Immunofluorescence}

For double immunostaining of SP and intrinsic factor, replicate sections were incubated with murine monoclonal IgM anti-SP $(1: 100)$ and rabbit polyclonal IgG anti-intrinsic factor $(1: 3,000)$ overnight at $4^{\circ} \mathrm{C}$. This was followed by incubation with donkey Cy2-labeled anti-rabbit IgG and donkey Cy3-labeled anti-mouse $\lg \mathrm{M}$.

\section{Statistics}

Comparison of parietal cell densities was performed by analysis of variance with post hoc comparison of significant means by Tukey's test. For all other comparisons, a Fisher exact test was used.

\section{Acknowledgements}

The authors thank Drs. Nicholas Wright and David Alpers for the generous gifts of antibodies used in these studies.

\section{References}

Dahm K and Werner B (1976). Susceptibility of the resected stomach to experimental carcinogenesis. Z Krebsforsch Klin Onkol Cancer Res Clin Oncol 85:219-229.

Dempsey PJ, Goldenring JR, Soroka CJ, Modlin IM, McClure RW, Lind CD, Ahlquist DA, Pittlekow MR, Lee DC, Sandgren EP, Page DL, and Coffey RJ (1992). Possible role of TGF $\alpha$ in the pathogenesis of Ménétrier's Disease: Supportive evidence from humans and transgenic mice. Gastroenterology 103:1950-1963.

Fox JG, Li X, Cahill RJ, Andrutis K, Rustgi AK, Odze R, and Wang TC (1996). Hypertrophic gastropathy in Helicobacter felis-infected wild-type C57BL/6 and p53 hemizygous transgenic mice. Gastroenterology 110:155-166.

Furukawa H, Iwanaga T, Hiratsuka M, Imaoka S, Ishikasa O, Kabuto T, Sasaki Y, and Kameyama M (1993). Gastric remnant cancer as a metachronous multiple lesion. Br J Surg $80: 54-56$. 
Goldenring JR, Ray GS, Coffey RJ, Meunier PC, Haley PJ, Barnes TB, and Car BD (2000). Reversible drug-induced oxyntic atrophy in rats. Gastroenterology 118:1080-1093.

Kaminishi M, Sadatsuki H, Johjima Y, Oohara T, and Kondo $Y$ (1987). A new model for production of chronic gastric ulcer by duodenogastric reflux in rats. Gastroenterology 92:19131918.

Kaminishi M, Shimizu N, Shimoyama S, Yamaguchi H, Ogawa T, Sakai S, Kuramoto S, and Oohara T (1995). Etiology of gastric remnant cancer with special reference to the effects of denervation of the gastric mucosa. Cancer 75:1490-1496.

Kaminishi M, Shimizu N, Shimoyama S, Yamaguchi H, Tsuji E, Aoki F, Nomura S, Yoshikawa A, Kuramoto S, Oohara T, Inada K, and Tatematsu M (1997). Denervation promotes the development of cancer-related lesions in the gastric remnant. J Clin Gastroenterol 25(Suppl):129-134.

Kaminishi M, Shimizu N, Yamaguchi $\mathrm{H}$, Hashimoto M, Sakai S, and Oohara T (1996). Different carcinogenesis in the gastric remnant after gastrectomy for gastric cancer. Cancer 77:1646-1653.

Karam SM and Leblond CP (1993). Dynamics of epithelial cells in the corpus of the mouse stomach. III. Inward migration of the neck cells followed by progressive transformation in to zymogenic cells. Anat Rec 236:297-313.

Kondo K, Suzuki H, and Nagayo T (1984). The influence of gastro-jejunal anastomosis on gastric carcinogenesis in rate. Gann 75:362-369.

Langhans P, Heger RA, Hohenstein J, and Bunte H (1981). Gastric stump carcinoma: New aspects deduced from experimental results. Scand J Gastroenterol Suppl 67:161-164.

Lee A, Fox JG, Otto G, and Murphy J (1990). A small animal model of human Helicobacter pylori active chronic gastritis. Gastroenterology 99:1315-1323.

Mason R and Filipe I (1990). The aetiology of gastric stump carcinoma in the rat. Scand J Gastroenterol 25:961-965.
Mason RC, Taylor PR, Filipe MI, and McColl I (1988). Pancreaticoduodenal secretions and the genesis of gastric stump carcinoma in the rat. Gut 29:830-834.

Ritchie WP (1984). Alkaline reflux gastritis: A critical reappraisal. Gut 25:975-987.

Salmon RJ, Merle S, Zafrani B, Decosse JJ, Sherlock P, and Deschner EE (1985). Gastric carcinogenesis induced by $\mathrm{N}$-methyl-N'-nitro-N-nitrosoguanidine: Role of gastrectomy and duodenal reflux. Jpn J Cancer Res 76:167-172.

Schmidt PH, Lee JR, Joshi V, Playford RJ, Poulsom R, Wright NA, and Goldenring JR (1999). Identification of a metaplastic cell lineage associated with human gastric adenocarcinoma. Lab Invest 79:639-646.

Szentleleki K, Morvay K, Pinter A, Borzsony M, Nagy L, Jahasz F, and Solyom C (1990). The extent of bile reflux and development of gastric cancer after resections in rat. Acta Chir Hung 31:339-346.

Wang TC, Dangler CA, Chen D, Goldenring JR, Koh T, Raychowdhury R, Coffey RJ, Ito S, Varro A, Dockray GJ, and Fox JG (2000). Synergistic interaction between hypergastrinemia and Helicobacter infection in a mouse model of gastric cancer. Gastroenterology 118:36-47.

Wang TC, Goldenring JR, Dangler C, Ito S, Mueller A, Jeon WK, Koh TJ, and Fox JC (1998). C57BL/6 mice deficient in secretory phospholipase A2 show increased apoptosis and altered cellular differentiation during Helicobacter felis infection. Gastroenterology 114:675-689.

Wright NA, Pike C, and Elia G (1990). Induction of a novel epidermal growth factor-secreting cell lineage by mucosal ulceration in human gastrointestinal stem cells. Nature 343: 82-85.

Yamaguchi $\mathrm{H}$, Goldenring JR, Kamanichi M, and Lee JR (2002). Identification of spasmolytic polypeptide expressing metaplasia in remnant gastric cancer and surveillance postgastrectomy biopsies. Dig Dis Sci 47:573-578. 